\title{
A search for evolved dust in Herbig Ae stars
}

\author{
A. Natta ${ }^{1}$, L. Testi ${ }^{1}$, R. Neri ${ }^{2}$, D. S. Shepherd ${ }^{3}$, and D. J. Wilner ${ }^{4}$ \\ 1 Osservatorio Astrofisico di Arcetri, INAF, Largo E.Fermi 5, 50125 Firenze, Italy \\ 2 IRAM, 300 rue de la Piscine, 38406 St Martin d'Heres, France \\ 3 National Radio Astronomy Observatory, PO Box O, Socorro, NM 87801, USA \\ ${ }^{4}$ Harvard-Smithsonian Center for Astrophysics, 60 Garden Street, Cambridge, MA 02138, USA
}

Received 3 November 2003 / Accepted 24 November 2003

\begin{abstract}
We present observations of six isolated, pre-main-sequence, intermediate mass stars selected for shallow spectra at submillimeter wavelengths at 1.3, 2.6, 7.0, and 36 millimeters from the IRAM PdBI and the VLA. We analyze the new observations of these stars (HD 34282, HD 35187, HD 142666, HD 143006, HD 150193, HD 163296) together with similar observations of three additional stars from the literature (CQ Tau, UX Ori, TW Hya), in the context of self-consistent irradiated disk models. Our aim is to constrain the wavelength dependence of the dust opacity and the total dust mass in the disks. The shallow wavelength dependence of the opacity is confirmed and for a few stars extended to significantly longer wavelengths. For any plausible dust properties, this requires grain growth from interstellar sizes to maximum sizes of at least a few millimeters, and very likely to several centimeters or more. For four of the stars (HD 34282, HD 163296, CQ Tau, TW Hya), the millimeter emission has been spatially resolved, and the large disk radii (>100 AU) rule out that high optical depths play a role. The mass of dust that has been processed into large grains is substantial, and in some cases implies a disk mass comparable to the mass of the central star.
\end{abstract}

Key words. stars: planetary systems: protoplanetary disks - stars: planetary systems: formation - stars: formation

\section{Introduction}

In the dense environment of circumstellar disks, grains are subject to coalescence and fragmentation, which will, in time, alter their properties and very likely cause significant growth from the sub-micron size typical of dust in the diffuse interstellar medium (ISM). This is a necessary step for all theories of planet formation. However, the interplay of a multiplicity of poorly known physical processes and their dependence on details of the grain structure make it difficult to reach consensus on the extent and timescale of dust processing (see Beckwith et al. 2000 and references therein). It is therefore important to derive constraints from the observations, determining the properties of dust in disks surrounding pre-main-sequence stars of well known ages.

The spectral energy distribution (SED) of T Tauri stars at millimeter and submillimeter wavelengths has a shallow dependence on wavelength, consistent with optically thin emission from millimeter-size grains (Beckwith \& Sargent 1991). However, this interpretation of the SEDs has remained controversial, since it was soon realized that it is not possible to infer the dust opacity law from the SED alone, and that the effect of potentially large optical depth needs to be sorted out. To do that, one needs to combine the determination of the longwavelength SED with spatially resolved images of the disk at

Send offprint requests to: A. Natta,

e-mail: natta@arcetri.astro.it one (or possibly more) wavelengths (see e.g. the discussion in Testi et al. 2001). Recently, this has been done for two premain-sequence stars, TW Hya (Calvet et al. 2002) and CQ Tau (Testi et al. 2003), using the VLA array at $7 \mathrm{~mm}$ to resolve the disk emission. In both cases, it was found that the dust opacity depends on wavelength roughly as $\lambda^{-0.6}$, and this was interpreted as evidence for grain growth to centimeter sizes.

Following our work on CQ Tau, we have started a search for pre-main-sequence stars of intermediate mass with shallow SEDs extending to $7 \mathrm{~mm}$. The choice of this rather long wavelength was motivated by the advantages of using the largest wavelength range for the determination of the spectral index and by the fact that the VLA offers the best resolution available today, provided that the source is strong enough. In addition, the longer the wavelength the more severe the constraints on dust properties one can set.

We report in this paper the results for a sample of six stars. The sample and the observations obtained with the PdB and VLA interferometers are described in Sect. 2. The results are presented in Sect. 3 and their implications for grain properties and evolution are discussed in Sect. 4.

\section{Observations}

\subsection{Sample stars}

Our sample is composed by nine pre-main-sequence stars, whose properties are summarized in Table 1 (Cols. 1-7). 
Table 1. Star and disk properties.

\begin{tabular}{lccccccccccc}
\hline \hline Star $^{a}$ & ST & $\begin{array}{c}T_{\text {eff }} \\
(\mathrm{K})\end{array}$ & $\begin{array}{c}L_{\star} \\
\left(L_{\odot}\right)\end{array}$ & $\begin{array}{c}D \\
(\mathrm{pc})\end{array}$ & $\begin{array}{c}M_{\star} \\
\left(M_{\odot}\right)\end{array}$ & $\begin{array}{c}\text { Age } \\
(\mathrm{Myr})\end{array}$ & $\begin{array}{c}\lambda_{\max } \\
(\mathrm{mm})\end{array}$ & $\alpha_{\mathrm{mm}}$ & $\beta$ & $\begin{array}{c}M_{\mathrm{d}}^{\kappa b} \\
\left(M_{\odot}\right)\end{array}$ & $\beta_{\mathrm{SD}}$ \\
\hline$\underline{\mathrm{HD} \mathrm{34282}}$ & A0 & 9800 & 36 & 400 & 2.3 & 3 & 3.2 & 3.0 & 1.3 & 0.09 & 1.3 \\
$\mathrm{HD} \mathrm{150193}$ & A1 & 9500 & 21 & 160 & 2.3 & 6 & 7.0 & 4.0 & 1.6 & 0.02 & 1.0 \\
$\underline{\mathrm{HD} \mathrm{163296}}$ & A1 & 9500 & 36 & 122 & 2.3 & 5 & 7.0 & 2.6 & 0.8 & 0.05 & 1.0 \\
HD 35187 & A2 & 9100 & 34 & 150 & 2.2 & 5 & 3.6 & 2.6 & 0.7 & 0.003 & 1.5 \\
UX Ori & A3 & 8600 & 42 & 450 & 2.2 & 3 & 7.0 & 2.0 & 0.0 & 0.03 & 0.1 \\
HD 142666 & A8 & 7600 & 8 & 116 & 1.6 & $>10$ & 7.0 & 2.2 & 0.4 & 0.01 & 0.7 \\
CQ Tau & F2 & 6900 & 4 & 100 & 1.5 & $>10$ & 7.0 & 2.4 & 0.5 & 0.02 & 0.7 \\
HD 143006 & G6 & 5770 & 0.8 & 82 & 1 & $>10$ & 3.1 & 2.5 & 0.8 & 0.005 & 1.2 \\
TW Hya & K8 & 4000 & 0.23 & 55 & 0.6 & 10 & 7.0 & 2.3 & 0.7 & 0.03 & 0.7 \\
\hline${ }^{a}$ Disks resolved at millimeter wavelengths are underlined. & & & & & \\
${ }^{b} M_{\mathrm{d}}^{\kappa}=M_{\mathrm{d}} \times\left(\kappa_{1 \mathrm{~mm}} / 0.01\right)$.
\end{tabular}

Six of the stars listed are new observations, while three are from the literature (Testi et al. 2001, 2003; Calvet et al. 2002). The six new stars were selected because they were known to have shallow spectra at sub-millimeter or millimeter wavelengths from previous observations (Sylvester et al. 1996; Mannings \& Sargent 1997, 2000). We adopted distances and spectral types from Hipparcos and computed the stellar parameters (effective temperature and luminosity) as in Natta et al. (2000). Slight differences from the values obtained by other authors are within the uncertainties; they are not significant for the purposes of this paper. For HD 34282, we have adopted the recent estimate of the distance and luminosity of Piétu et al. (2003). HD 35187 is a binary with separation of $1.38^{\prime \prime}$; following Dunkin \& Crawford (1998), we have attributed the disk emission to the primary component HD 35187B. HD 143006 has no measured Hipparcos distance; we have adopted the distance of 82 pc estimated by Sylvester et al. (1996), as well as their spectral type.

Stellar masses and ages are estimated from the location of the stars on the HR diagram, using Palla \& Stahler (1993) evolutionary tracks. The stars in the sample are all relatively old objects ( $>$ few million years), as expected given their "isolated" nature. They cover a range of masses from about 1 to 2.3 solar masses, and luminosities from 0.23 to $42 L_{\odot}$.

\subsection{VLA observations}

New NRAO ${ }^{1}$ VLA $7 \mathrm{~mm}$ and $3.6 \mathrm{~cm}$ observations of HD 34282, HD 35187, HD 142666, HD 143006, HD 150193 , and HD 163296 (for the latter only $7 \mathrm{~mm}$ data was obtained) were performed on several occasions from November 2001 to May 2003. The array was mainly used in the most compact $\mathrm{D}$ configuration, offering baselines from the shadowing limit to $\sim 350 \mathrm{~m}$; the corresponding angular resolution at $7 \mathrm{~mm}$ is 2 arcsec. HD 142666, HD 143006, HD 150193, and HD 163296 were also observed in the C configuration,

\footnotetext{
${ }^{1}$ The National Radio Astronomy Observatory is a facility of the National Science Foundation operated under cooperative agreement by Associated Universities, Inc.
}

Table 2. VLA observation summary

\begin{tabular}{lcccc}
\hline \hline Star & $\alpha(\mathrm{J} 2000)$ & $\delta(\mathrm{J} 2000)$ & $\begin{array}{c}\lambda \\
(\mathrm{mm})\end{array}$ & $\begin{array}{c}F_{v} \\
(\mathrm{mJy})\end{array}$ \\
\hline HD 34282 & $05: 16: 00$ & $-09: 48: 35.4$ & 36 & $<0.2^{a}$ \\
& & & 7 & $<1^{a}$ \\
HD 35187 & $05: 24: 01$ & $24: 57: 37.6$ & 36 & $1.0 \pm 0.03$ \\
& & & 7 & $1.0 \pm 0.2$ \\
HD 142666 & $15: 56: 40$ & $-22: 01: 40.0$ & 36 & $<0.2^{a}$ \\
& & & 7 & $1.7 \pm 0.2$ \\
HD 143006 & $15: 58: 37$ & $-22: 57: 15.0$ & 36 & $<0.15^{a}$ \\
& & & 7 & $<0.7^{a}$ \\
HD 150193 & $16: 40: 18$ & $-23: 53: 45.2$ & 36 & $0.2 \pm 0.02$ \\
& & & 7 & $0.7 \pm 0.2$ \\
HD 163296 & $17: 56: 21$ & $-21: 57: 21.9$ & $61^{b}$ & $0.25 \pm 0.04$ \\
& & & $36^{b}$ & $0.42 \pm 0.025$ \\
& & & $13^{b}$ & $0.9 \pm 0.25$ \\
& & & 7 & $6 \pm 0.5$ \\
\hline
\end{tabular}

${ }^{a}$ Upper limits are $3 \sigma ;{ }^{b}$ Data obtained from VLA Archive, originally observed by Bouwman et al. (2000).

with baselines up to $3.4 \mathrm{~km}$ and resolution $\sim 0.5$ arcsec. In both cases the largest angular scale that can be accurately imaged is $\sim 40^{\prime \prime}$. Calibration and editing of the $(u, v)$ data were performed using standard techniques within the AIPS package. Images were produced using the AIPS IMAGR task with natural weighting of the visibilities. The measured fluxes or upper limits are reported in Table 2. For HD 163296, there were previous data at centimeter wavelengths in the VLA archive (see also Bouwman et al. 2000); we have re-reduced them with the same procedure used for our new observations and added the resulting fluxes to Table 2. Flux calibration was performed using standard techniques at 3.6 and $0.7 \mathrm{~cm}$ observing $3 \mathrm{C} 286$ and/or 3C48 and deriving the flux density at the time of observations for the phase calibrators. This procedure is expected to be accurate within $1 \%$ at $3.6 \mathrm{~cm}$ and within $\sim 15 \%$ at $0.7 \mathrm{~cm}$. 
Table 3. PdB observation summary.

\begin{tabular}{lcccc}
\hline \hline Star & $\alpha(\mathrm{J} 2000)$ & $\delta(\mathrm{J} 2000)$ & $\begin{array}{c}\lambda \\
(\mathrm{mm})\end{array}$ & $\begin{array}{c}F_{v} \\
(\mathrm{mJy})\end{array}$ \\
\hline HD 34282 & $05: 16: 00$ & $-09: 48: 35.4$ & 3.2 & $5.5 \pm 1$ \\
& & & 1.3 & $100 \pm 23$ \\
HD 35187 & $05: 24: 01$ & $24: 57: 37.6$ & 3.6 & $2.5 \pm 0.4$ \\
& & & 1.3 & $20 \pm 2$ \\
HD 142666 & \multirow{2}{*}{$15: 56: 40$} & $-22: 01: 40.0$ & 3.3 & $11 \pm 1$ \\
& & & 3.1 & $13 \pm 1$ \\
& & & 1.2 & $79 \pm 4$ \\
HD 143006 & \multirow{2}{*}{$15: 58: 37$} & $-22: 57: 15.0$ & 3.1 & $4.6 \pm 0.7$ \\
& & & 1.2 & $43 \pm 3$ \\
\hline
\end{tabular}

\subsection{Plateau de Bure observations}

Observations of four of our target stars were made simultaneously at $\sim 3 \mathrm{~mm}$ and $1.2 \mathrm{~mm}$ using the Plateau de Bure interferometer $^{2}$ on May 18 and 29, 2001 and August 18 and 19, 2001. Visibilities were obtained in the most compact configuration of the 5 antenna array, yielding projected baselines which range from about $64 \mathrm{~m}$ down to the antenna diameter of $15 \mathrm{~m}$. The largest angular scales that can be imaged range from $\sim 20^{\prime \prime}$ at $3.5 \mathrm{~mm}$ to less than $8^{\prime \prime}$ at $1.2 \mathrm{~mm}$. The $46^{\prime \prime}\left(20^{\prime \prime}\right.$ at $\left.1.2 \mathrm{~mm}\right)$ primary beam field of the interferometer was centered at the nominal position of the each star (see Table 3 ).

At $1.2 \mathrm{~mm}$, data were taken in double sideband mode, while at $\sim 3 \mathrm{~mm}$ observations were made in upper sideband only (see Table 3 for the tuning frequencies).

Data calibration was performed in the antenna-based manner. Flux densities were obtained from the visibilities using standard IRAM fitting procedures. The calibration is expected to have an uncertainty of $\sim 15 \%$ at $3 \mathrm{~mm}$ and $\sim 20 \%$ at $1.2 \mathrm{~mm}$. All the sources turned out to be unresolved; the derived fluxes are given in Table 3 .

\section{Results}

Figure 1 shows the observed fluxes as function of wavelength for each star. PdB and VLA detections are shown as filled dots; the errors are smaller than the symbol size. Arrows are $3 \sigma$ upper limits.

We show in Fig. 1 also interferometric data from the literature, obtained with PdB (Piétu et al. 2003) and OVRO (Manning \& Sargent 1997; 2000), as well as single-dish fluxes measured with the JCMT by Sylvester et al. (1996) and Mannings (1994). At about $1 \mathrm{~mm}$, where the two sets of data overlap, the single-dish fluxes tend to be larger than the interferometric ones by factors of order 1.3-1.6, somewhat larger than the calibration errors (which are of the order of 20\%). Note however that this is not always the case, e.g. HD 34282 where the single-dish and interferometric fluxes agree within the errors. It is likely that this difference is caused by calibration uncertainties and by the different width of the interferometer and single-dish bolometer bandpass, but it is also possible that the

${ }^{2}$ The Plateau de Bure interferometer at IRAM is supported by INSU/CNRS (France), MPG (Germany) and IGN (Spain). interferometers are missing some diffuse emission. We will go back to this point in Sect. 3.3.

\subsection{Radio fluxes and gas emission}

The main purpose of our $3.6 \mathrm{~cm}$ observations was to check for contamination of the millimeter dust emission by free-free or non-thermal emission. Three objects in our sample were not detected at $3.6 \mathrm{~cm}$ : HD 34282, HD 142666 and HD 143006; two of these (HD 34282 and HD 143006) were not detected at $7 \mathrm{~mm}$ either. The upper limits shown in Fig. 1 indicate that at millimeter wavelengths there should be very little contribution from gas emission.

Three objects were detected. HD 35187 is a very strong radio source; the spectral index between $7 \mathrm{~mm}$ and $3.5 \mathrm{~cm}$ is $\sim 0$. It is possible that we have detected a radio flare; in any case, we have made the conservative assumption that only a negligible fraction of the observed $7 \mathrm{~mm}$ flux is due to dust, and evaluated the ionized gas contribution to shorter wavelength as a power-law of slope -0.1 (dashed line in Fig. 1).

HD 150193 has a rather steep slope between $7 \mathrm{~mm}$ and $3.5 \mathrm{~cm}$. We have assumed that the $3.5 \mathrm{~cm}$ flux is free-free emission from a wind, for which we expect a spectral slope of $\sim 0.6$ (Felli \& Panagia 1981). The dashed line in Fig. 1 shows the estimated gas emission; it accounts for $\sim 70 \%$ of the observed 7 mm flux.

In the case of HD 163296 there are additional radio observations at $1.3 \mathrm{~cm}$ and $6 \mathrm{~cm}$ (Bouwman et al. 2000). The three centimeter-wavelength fluxes are consistent with wind emission with slope $\sim 0.6$ (dashed line in Fig. 1); the corresponding contribution at $7 \mathrm{~mm}$ is negligible.

\subsection{Dust emission}

Table 1, Col. 9 shows the spectral index of the dust emission $\alpha_{\mathrm{mm}}\left(F_{v} \propto v^{\alpha_{\mathrm{mm}}}\right)$, after subtraction of the free-free component, measured between $1.3 \mathrm{~mm}$ and a maximum wavelength (either 2.6 or $7.0 \mathrm{~mm}$ ) given in Col. 8. The error on $\alpha_{\mathrm{mm}}$ is about \pm 0.2 and is dominated by the systematic uncertainties on the flux scale at different wavelengths and telescopes. In all objects but one $\alpha_{\mathrm{mm}} \lesssim 3$. The exception is HD 150193, which has no interferometric measurement at $1.3 \mathrm{~mm} ; \alpha_{\mathrm{mm}}$ is computed between 2.6 and $7 \mathrm{~mm}$, and is therefore much more uncertain than in the other objects.

\subsection{Disk models}

Relatively flat spectral indices at millimeter wavelengths can be the signature of optically thin emission from grains whose opacity has a very shallow dependence on wavelength, but also of optically thick emission from grains of any kind. Once all other parameters are fixed, it is possible to obtain shallower and shallower values of $\alpha_{\mathrm{mm}}$ down to the limit $\alpha_{\mathrm{mm}}=2$ by increasing the disk mass (and therefore making the dust emission optically thick). However, at any given wavelength the corresponding flux will increase to very large values, unless the disk 


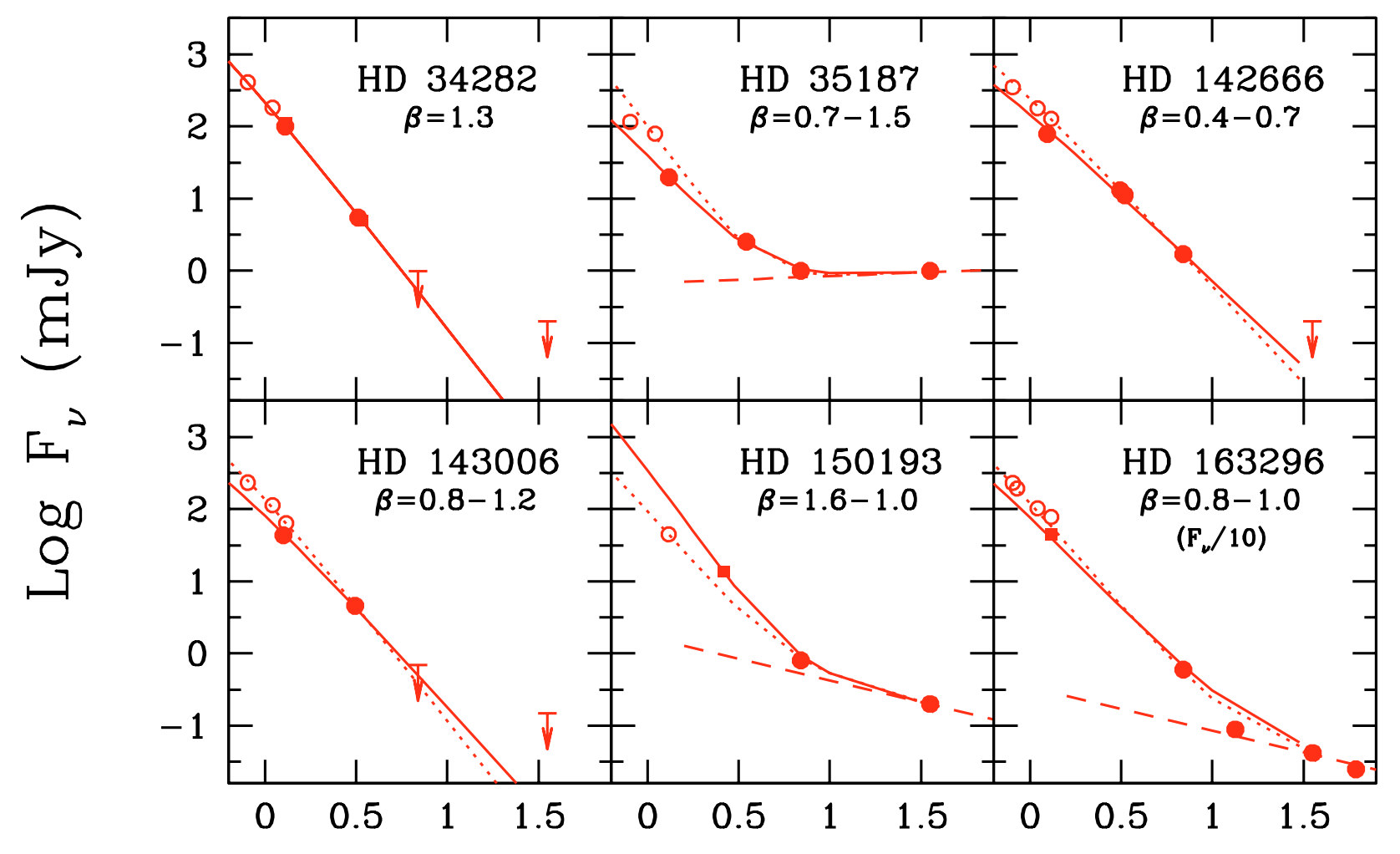

\section{$\log \lambda(\mathrm{mm})$}

Fig. 1. Observed fluxes for the six stars in our sample. Filled dots and arrows show detections and $3 \sigma$ upper limits from this paper (PdB and VLA); filled squares are interferometric data from Piétu et al. (2003; HD 34282, nearly coincident with our points) and Mannings \& Sargent (1997; HD 150193 and HD 163296), respectively. Open dots are single dish JCMT data (Sylvester et al. 1996; Mannings 1994). Dashed lines show the adopted fit to the centimeter fluxes. The solid lines show the results of disk models, to which we have added the estimated free-free contribution (see text). Dotted lines show disk models that fit the single-dish fluxes (rather than the interferometric ones) at $\lambda \leq 1.3 \mathrm{~mm}$. The corresponding values of $\beta$, the opacity power-law exponent, are shown on each panel; the first value has been derived fitting our interferometric data at all wavelengths $(\beta$ in the text and in Table 2$)$, the second using single-dish data for $\lambda \leq 1.3 \mathrm{~mm}\left(\beta_{\mathrm{SD}}\right.$ in the text and in Table 2). Note that for HD 163296 the models overpredict the $1.3 \mathrm{~cm}$ flux; this happens because we have assumed that the dust opacity is an unbroken power-law to $\lambda=3 \mathrm{~cm}$, which is likely not the case.

is very small and highly tilted to the line of sight (see the discussion in Testi et al. 2001).

A good illustration is the case of UX Ori, which has the flattest spectral index so far $\left(\alpha_{\mathrm{mm}}=2.03 \pm 0.26\right.$; Testi et al. 2001). The observations could be reproduced by disks with ISM grains if $R_{\mathrm{d}}=30 \mathrm{AU}, \theta=66^{\circ}, M_{\mathrm{d}} \sim 2 M_{\odot}$. Larger disks need to be more tilted ( $\theta=86^{\circ}$ for $R_{\mathrm{d}}=100 \mathrm{AU}$, for example), obscuring the central star, and even more massive, well above the limit where disks become unstable to self-gravity. If the disk is large (say, >100 AU), then it must be optically thin at millimeter wavelengths; the observed $\alpha_{\mathrm{mm}}$ can only be reproduced if the dust opacity is practically independent of wavelengths.

We have analyzed the stars in this sample following the same procedure already used for UX Ori and CQ Tau by Testi et al. (2001). We derive the dust opacity law by comparing for each star the observed fluxes, corrected for free-free contamination, to the predictions of self-consistent disk models. We use the two-layer models of flared disks (i.e., in hydrostatic equilibrium) heated by stellar irradiation as developed by Dullemond et al. (2001), following the schematization of
Chiang \& Goldreich (1997). These models have been used in the analysis of CQ Tau by Testi et al. (2003) and we refer to that paper for a more detailed description.

To characterize completely such a disk model, once the stellar properties are known, one needs to specify a number of parameters, namely the inclination $\theta$ with respect to the observer $(\theta=0$ for face-on disks), the disk inner and outer radii ( $R_{\text {in }}$ and $R_{\mathrm{d}}$ ), the disk mass $\left(M_{\mathrm{d}}\right)$, the dependence of the surface density on radius $\left(\Sigma \propto R^{-p}\right)$, the properties of dust on the disk surface and in the midplane. However, millimeter fluxes are practically independent of some of these parameters, for example the disk inner radius and the surface dust properties. Also, they are insensitive to most details of the adopted radiation transfer scheme (see Dullemond \& Natta 2003).

For each star we have computed a large grid of models, varying $R_{\mathrm{d}}, M_{\mathrm{d}}, p, \theta$ and the dust opacity in the midplane, described by a power-law $\kappa=\kappa_{1} \mathrm{~mm}(\lambda / 1 \mathrm{~mm})^{-\beta}$ with $\kappa_{1 \mathrm{~mm}}=0.01 \mathrm{~cm}^{2} \mathrm{~g}^{-1}$ as a fiducial value (which includes the gas contribution to the total mass for a gas-to-dust mass ratio 100). $R_{\text {in }}$ is fixed at the dust sublimation radius; for the properties of 
the dust on the disk surface we have adopted the same model as Testi et al. (2003); neither of these model parameters is constrained by our data, nor do these choices affect the interpretation of the millimeter spectra.

For two of the stars in the sample, the millimeter observations resolve the disk emission and provide lower limits on $R_{\mathrm{d}}$. HD 34282 has been resolved at $1.3 \mathrm{~mm}$ by Piétu et al. (2003), who measure a deconvolved FWHM size of $1.74 \pm 0.07^{\prime \prime} \times$ $0.89 \pm 0.06^{\prime \prime}$ and derive an inclination of $56 \pm 3 \mathrm{deg}$. Note that the fluxes measured by Piétu et al. (2003) agree with our measurements to within the errors. Since the deconvolved FWHM size is a lower limit to the physical size (Dutrey et al. 1996; Piétu et al. 2003; Testi et al. 2003), at a distance of 400 pc, the outer disk radius must be $R_{\mathrm{d}}>350 \mathrm{AU}$. With this lower limit to $R_{\mathrm{d}}$ and the observed $\theta$, our models fit the observed fluxes only if the disk is optically thin at millimeter wavelengths. Thus, the only quantities that one can derive from the data are $\beta$ and the disk mass for the fiducial value of $\kappa_{1 \mathrm{~mm}}$, that we will call $M_{\mathrm{d}}^{\kappa}=M_{\mathrm{d}} \times\left(\kappa_{1 \mathrm{~mm}} / 0.01\right)$. The results are $\beta \sim 0.7$ and $M_{\mathrm{d}}^{\kappa} \sim 0.1 M_{\odot}$, respectively. The uncertainties are typically about \pm 0.1 for beta and a factor of 2 for $M_{\mathrm{d}}^{\kappa}$ (for $p=1-2$ ), as discussed in Testi et al. (2003). They are due to the fact that the surface density profile is not well constrained by the data, and it is possible to reproduce the same intensity map with different values of $p$ by changing $R_{\mathrm{d}}$ (the larger $R_{\mathrm{d}}$, the steeper the density profile one needs). Note that for HD 34282 the value of $\beta$ would be the same if the distance were $D=160 \mathrm{pc}$, as measured by Hipparcos, but the disk mass would be reduced by a factor $\sim 6$.

HD 163296 is partially resolved in $1.3 \mathrm{~mm}$ continuum emission by Mannings \& Sargent (1997), who measure a deconvolved FWHM size of $110 \times 95 \mathrm{AU}$; in the CO (2-1) line the disk size is $310 \times 160 \mathrm{AU}$. Our $7 \mathrm{~mm}$ observations with the VLA in C configuration indicate that the emission is resolved with deconvolved size about $300 \times 180 \mathrm{AU}$ and inclination $55^{\circ}$ (Testi et al. 2004). As soon as $R_{\mathrm{d}} \gtrsim 100 \mathrm{AU}$, the millimeter emission is optically thin with $\beta \sim 0.8$ and $M_{\mathrm{d}}^{\kappa} \sim 0.05 M_{\odot}$.

To the best of our knowledge, none of the other disks have been resolved at any wavelength, and we have no indication of the disk outer radius. However, we consider it unlikely that they are all very small, massive disks, seen almost edge-on, as one would require to reproduce the observations if the grains are as in the ISM. We have therefore proceeded assuming $R_{\mathrm{d}}>100 \mathrm{AU}$ and allowing the other disk parameters to vary, including the dust opacity. The resulting values of $\beta$ and $M_{\mathrm{d}}^{K}$ are shown in Table 1, Cols. 9 and 10, respectively. Note that a small, truncated disk may be more likely in HD 35187, which is a binary with separation of 200 AU. Still, a disk with $R_{\mathrm{d}} \sim 70 \mathrm{AU}$ (i.e., $1 / 3$ the separation) reproduces the observed fluxes only if it is optically thin.

The unresolved fluxes analyzed here do not constrain the value of $p$, since it is generally possible to fit the SED data with either very flat $(p=0.5)$ or very steep $(p=2)$ surface density profiles. Increasing $p$ increases the contribution to the millimeter fluxes of the inner, optically thick disk, whose emission does not depend on $\beta$, and we may wonder how much mass can be "hidden" in this region. However, we find that $M_{\mathrm{d}}^{K}$ is a rather good estimate of the total mass of the grains that contribute to the millimeter-wavelength opacity. All available high angular resolution observations suggest that $p \leq 1.5$ (Dutrey et al. 1996; Wilner et al. 2000; Kitamura et al. 2002; Testi et al. 2003). Typically, in disks with flat surface density profile ( $p \lesssim 1.5$ ), the inner, optically thick (at $\sim 1.2 \mathrm{~mm}$ ) part of the disk contains only about $10 \%$ of the total mass.

One final potential source of uncertainties on our estimates of $\beta$ stems from the characteristics of the interferometers themselves, which filter out any emission more extended than the largest angular scale mentioned in Sect. 2.2; in particular, at $1.2 \mathrm{~mm}$, this effect could be important. To estimate the effect of this potential problem, we have computed values of $\beta\left(\beta_{\mathrm{SD}}\right)$ fitting the single-dish fluxes at $\lambda \leq 1.3 \mathrm{~mm}$ and the longer wavelength interferometric points (Col. 11). This combination gives a steeper spectral slope, and a correspondingly higher value of $\beta$. This value is likely to be largely overestimated. In fact, if there is a diffuse overlying emission component, this should also be present at longer wavelengths, and by combining in this way single-dish and interferometer data we are biasing our results toward steeper spectral slopes. Additionally, if diffuse emission is indeed present, it is unlikely to come from the disk itself, so that we should in any case exclude it when fitting disk models to the data. In spite of these considerations, one shoud keep in mind the possibility that the interferometric fluxes are somewhat underestimated, and the values of $\beta_{\mathrm{SD}}$ provide a rough estimate of the observational uncertainties in the derivation of $\beta$.

\section{Discussion}

The results of our analysis are summarized in Table 1. To the six stars in our sample, we have added UX Ori and CQ Tau (data from Testi et al. 2001, 2003 and references therein) and TW Hya (data from Weintraub et al. 1989; Wilner et al. 2000, 2003 and references therein), and we have reanalyzed them again in the same manner for homogeneity. The objects that have been spatially resolved are underlined. In all the objects the values of $\beta$ are well below 2, which is the typical wavelength dependence of the opacity of small ISM grains. This is true also if we consider the upper limits $\beta_{\mathrm{SD}}$. For the four resolved systems, the disk sizes are such that optically thick emission is not a viable possibility (see Sect. 3.2 and Testi et al. 2001, 2003; Calvet et al. 2002). There is no doubt that in these objects the grains have been hugely processed. The remaining five systems have not been resolved; however, as discussed in Sect. 3.2, although the values of $\beta$ we derive for these objects are more uncertain (because the observations do not exclude an optically thick contribution to the emission), it is very unlikely that all of these stars have very compact disks with unprocessed ISM grains.

Once we have established that the grains originally present in the disk have undergone a large degree of processing, can we make the next step and derive the properties that characterize the actual grain population, for example the maximum grain size? This is not straightforward when considering the complexity added by a wide distribution of grain sizes and composition, as we will now illustrate. 


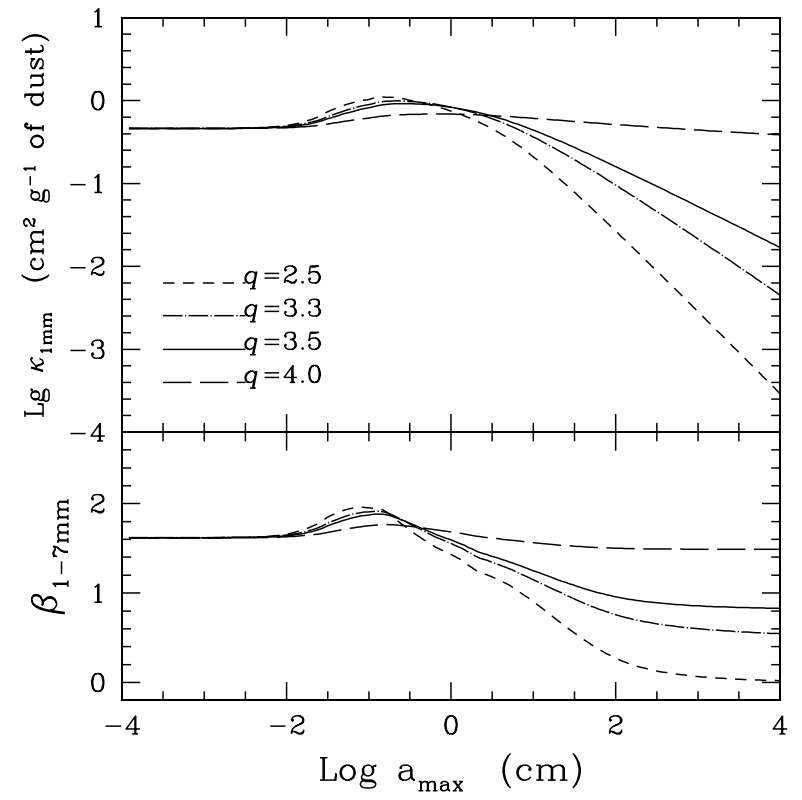

Fig. 2. Top panel: dust opacity at $1 \mathrm{~mm}$ as function of $a_{\max }$ for a size distribution $n(a) \propto a^{-q}$ between $a_{\min }=0.01 \mu \mathrm{m}$ and $a_{\max }$. Different curves are for different values of $q$, as labelled. The grains are porous conglomerates of $5 \%$ (in volume) olivine $\left(\left[\mathrm{Fe}_{0.3} \mathrm{Mg}_{0.7}\right]_{2} \mathrm{SiO}_{4}\right)$, $15 \%$ organic materials, $35 \%$ water ice and $50 \%$ vacuum; see http://www . astro. uni-jena.de/Users/dima/Opacities/RI/ new_ri.html for the cross sections of the individual components. Cross sections of the porous conglomerates have been computed as in Krügel \& Siebenmorgen (1994). Bottom panel: $\beta$ between $1 \mathrm{~mm}$ and $7 \mathrm{~mm}$ as function of $a_{\max }$ for the same grain distributions.

Let us consider a population of grains with a power-law size distribution $n(a) \propto a^{-q}$ between a minimum and a maximum size, $a_{\min }$ and $a_{\max }$, respectively. In the ISM, $a_{\min }$ is few tens of $\AA, a_{\max }$ is $\sim 0.1-0.2 \mu \mathrm{m}$, and $q=3.5$. These values will change if grains are processed in disks, and one can expect much larger values of $a_{\min }, a_{\max }$, and a variety of values of $q$. Small values of $q$ are expected when coagulation processes dominate, while large values of $q$ characterize fragmentation (see, for example, Weidenschilling 1997). For a given dust model, i.e., once the chemical structure, composition, porosity etc. are specified, one can compute the opacity for different values of $a_{\min }, a_{\max }$ and $q$. We choose for our discussion porous composite grains with $50 \%$ vacuum and state-of-the-art cross sections, as detailed in the caption of Fig. 2. The top panel shows the average dust opacity at $1 \mathrm{~mm}$ as function of $a_{\max }$ for various values of $q$; the bottom panel the corresponding values of $\beta$. The results do not depend on $a_{\min }$ as long as it is $\ll \lambda$.

The value of $\beta$ that characterizes the average opacity of a grain distribution decreases as $a_{\max }$ increases, as expected since grains with size $\gg \lambda$ have wavelength-independent opacity. However, only for $q<3, \beta$ goes to zero for large $a_{\max }$; for $q>3$, the small grains always contribute to the opacity, so that, as $a_{\text {max }}$ increases, $\beta$ reaches an asymptotic values that depends on $q$ and on the $\beta$ of the small grains. For $q=4$, the asymptotic value is practically that of the small grains.

Three objects in our sample (CQ Tau, TW Hya, HD 142666) have $\beta \sim 0.5-0.7$. This is consistent with $q \sim 2.5$ and $a_{\max } \sim 80 \mathrm{~cm}$; a distribution with $q=3.3$ has an asymptotic value $\beta=0.5$, and it is consistent with the observations as soon as $a_{\max }>300 \mathrm{~cm}$. In both cases the $1 \mathrm{~mm}$ opacity is much lower than our fiducial value, and the estimates of the disk mass needs to be raised by a factor $>40$ for $q=3.3$ and about 15 for $q=2.5$. The corresponding disk masses are high $(>0.3,0.9$, $0.3 M_{\odot}$, respectively); however, only in the case of TW Hya they violate the requirement that the disks must be gravitationally stable, i.e., $M_{\mathrm{d}} / M_{\star} \lesssim 30 \%$. Objects like HD 163296 have $\beta \sim 0.8-1$; they are consistent with $q=2.5, a_{\max } \sim 10 \mathrm{~cm}$, that would results in a disk mass $0.3 M_{\odot}$, or with any steeper size distribution with larger $a_{\max }$. A distribution with $q=3.6$ has an asymptotic value $\beta \sim 1$ for $a_{\max } \gtrsim 150 \mathrm{~cm}$, the corresponding disk mass would be $>0.5 M_{\odot}$. Very steep size distributions, such as $q=4$, never fit the observations.

The most extreme case in our sample, UX Ori (for which, however, there is no spatially-resolved disk image) has $\beta \sim 0$. If the disk is optically thin, this would require flat grain size distributions and very large $a_{\max }$; for example, $\beta=0.2$ corresponds to $q=2.5, a_{\max } \sim 10^{3} \mathrm{~cm}$. For such grain size distribution, however, the disk mass becomes $\sim 4 M_{\odot}$, i.e., $\gg M_{\star}$

These results depend on the dust model one adopts. For example, if the fraction of vacuum in the same grain model of Fig. 2 is reduced from $50 \%$ to $10 \%$, the value of $a_{\max }$ which reproduces the observations is reduced by a factor $\sim 3-8$ and $M_{\mathrm{d}}$ by a factor $2-3(q \leq 3.3)$. Grains of the same size and composition, but different structure and topology (homogeneous, composite aggregates, porous homogeneous or composite spherical particles, onion-shells particles, etc.) can have different values of $\beta$ and $\kappa_{1} \mathrm{~mm}$ (see, for example, Miyake \& Nakagawa 1993; Krügel \& Siebenmorgen 1994; Semenov et al. 2003 and the Jena web page), leading to rather different estimates of $a_{\max }$ and of the disk mass. Just to mention two examples of how all this affects the interpretation of the data, Testi et al. (2001), fitted the same UX Ori spectrum discussed above with ice-coated silicates of about $10 \mathrm{~cm}$ radius and a disk mass in the range 0.3-1 $M_{\odot}$, depending on the grain density. Calvet et al. (2002) obtained a good fit to the millimeter properties of TW Hya for $q=3.5, a_{\max } \sim 1 \mathrm{~cm}$ and $M_{\mathrm{d}} \sim 0.1 M_{\odot}$, using the dust model of Pollack et al. (1994) of compact segregate spheres where the relative fraction of water ice to organic materials was somewhat decreased (P. D'Alessio, private communication).

All this shows that it is not possible to disentangle from the value of $\beta$, which is just an average quantity, all the details that specify a given dust model. However, there are some basic grain properties that are constrained, since no realistic grain model results in $\beta<1$ for $a_{\max }<1 \mathrm{~mm}$. In fact, for most known grain mixtures, our observations are consistent with power-law grain size distributions where $a_{\max }$ is few ten to few hundred centimeters.

To account for the observed fluxes, the amount of mass in these grains has to be very large, implying that, at least when gas was present in the standard ISM ratio, the disk was close or above the limit for gravitational instability. Note that we have converted the solid mass into gas+dust mass assuming a gas-todust ratio of 100 . This conversion factor may not be appropriate for the present-time disk, if a significant fraction of the gas has evaporated from the system. Still, it provides a correct estimate 


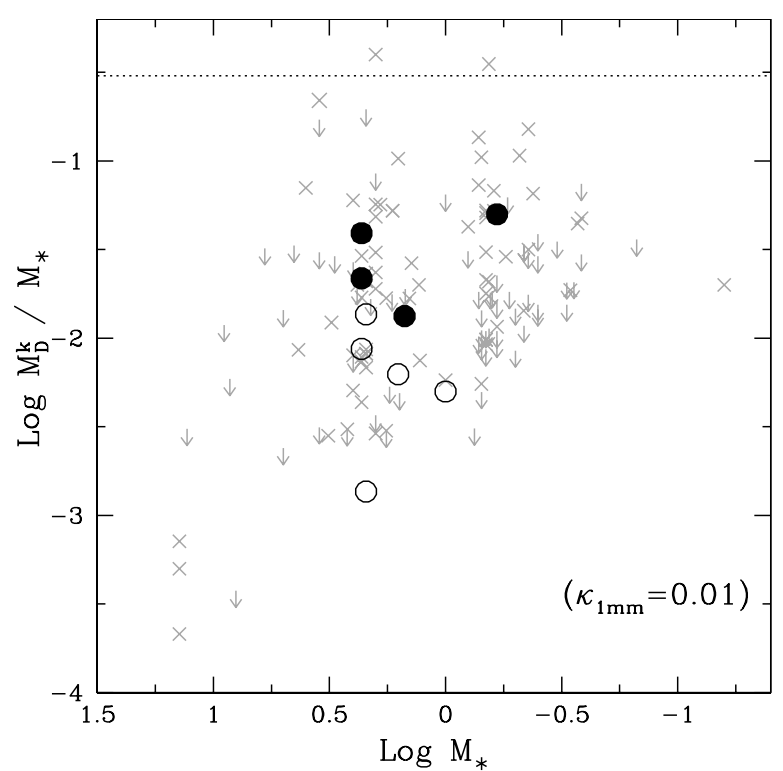

Fig. 3. Ratio of the disk to the stellar mass as function of $M_{\star}$ for a large sample of pre-main-sequence stars (adapted from Natta et al. 2000). Crosses are detections, upper limits are indicated with arrows. The objects studied in this paper are marked as circles; the four resolved ones are indicated with filled circles. All the disk masses have been computed for an opacity $\kappa_{1 \mathrm{~mm}}=0.01 \mathrm{~cm}^{2} \mathrm{~g}^{-1}$. The dotted line corresponds to $M_{\mathrm{d}} \times\left(\kappa_{1 \mathrm{~mm}} / 0.01\right) / M_{\star}=0.3$.

of the original disk mass, when the disk composition reflected that of the parent cloud. It is interesting that the value of $M_{\mathrm{d}}^{K}$ of our sample stars is similar to what is found for all the other premain-sequence stars studied so far (see Fig. 3). One wonders in how many cases the disk masses are underestimated by using $\kappa_{1 \mathrm{~mm}}=0.01 \mathrm{~cm}^{2} \mathrm{~g}^{-1}$.

Our data do not rule out the possibility that a non-negligible fraction of the original solid mass is in even larger bodies $(\mathrm{km}-$ size), from which planets can be formed. However, it seems unlikely that these could contain most of the solid mass, and that the millimeter-emitting grains were just the tail of the size distribution. If, for example, $99 \%$ of the solid mass is in kilometersize bodies, whose contribution to the millimeter opacity is negligible, their mass should be added to the values we have derived, making the disk mass 100 times larger.

An implicit assumption we have made so far is that the grain size distribution is continuous between $a_{\min }$ and $a_{\max }$. Models of planetesimal formation (Weidenschilling 1997) show the developement of a gap in the size distribution between $a_{1} \sim$ few centimeters and $a_{2} \sim$ few meters, which tends to fillin with time. In this case, one should compare $a_{\max }$ with $a_{1}$, and our results are consistent with these model predictions. Still, as we have seen, it is unlikely that most of the dust mass is on sizes $\gg a_{2}$, i.e., in bona-fide planetesimals, since the solid mass with sizes $<a_{1}$ is already very large (see Testi et al. 2003 for a more detailed discussion of this point). This is an interesting point, especially because all the stars in the sample are relatively old, $\gtrsim$ few million years, and in some case $\gtrsim 10$ million years. Most models of grain evolution have a very short timescale for the formation of planetesimals (e.g., Weidenschilling 2000 and references therein). One possibility is that the growth of kilometer-size bodies is in fact very inefficient, either because it is much slower than predicted or because it involves only a small fraction of the dust. Another intriguing possibility is that the formation of planetesimals takes place in a very early stage of the star formation, in very massive disks which are gravitationally unstable. However, a discussion of these points is well behond the scope of this paper.

\section{Conclusions}

This paper presents the results of a study of six pre-mainsequence, isolated, intermediate mass stars, performed with the PdB (at 1.3 and $2.6 \mathrm{~mm}$ ) and VLA (at $7 \mathrm{~mm}$ and $3.6 \mathrm{~cm}$ ) interferometers. All the stars were selected to have indications of flat spectral indexes from previous millimeter and submillimeter data. Our measurements confirm this indication, and extend our knowledge of the dust emission continuum down to $7 \mathrm{~mm}$. In all the objects $\alpha_{\mathrm{mm}} \lesssim 3$ for wavelengths between $\sim 1 \mathrm{~mm}$ and $2.6 \mathrm{~mm}$, and in 3 cases between $\sim 1 \mathrm{~mm}$ and $7 \mathrm{~mm}$.

We have added to these six stars two others (UX Ori and CQ Tau), previously studied in the same way by our group (Testi et al. 2001, 2003) and TW Hya, for which similar data exist in the literature (Calvet et al. 2002 and references therein).

The observations have been compared to the predictions of self-consistent irradiated disk models to derive the properties of the millimeter-emitting dust. As discussed by various authors, this cannot be done unambiguously if only the SED is known. To solve the ambiguity introduced by optical depth effects, one needs to spatially resolve the emission at some millimeter wavelength, or, at least, to have an estimate of the FWHM size. This additional information is available for two stars in our sample (HD 34282 and HD 163296), and for CQ Tau and TW Hya. For these objects, there is no doubt that grains in disks are heavily processed, causing a net growth, to maximum sizes that are certainly larger than few millimeters and, for the majority of grain models available in the literature, of at least few centimeters. The mass of the grains that have been thus processed is very large, in some cases comparable to the mass of the central star. Many grain size distributions are consistent with the data, but not very steep ones, $(q \gtrsim 4)$, unless micronsize grains have $\beta \lesssim 0.5-1$.

The uncertainty on $a_{\max }$ and $q$ reflects our ignorance on the details of the grain chemistry, structure and topology. However, in spite of these uncertainties, we are developing a view of the outer disks of pre-main-sequence stars as made of a huge mass of sand and pebbles, rather than of micron-size grains. Grain growth models can and should be tested against these observations.

Acknowledgements. We thank Endrik Krügel for having provided to us his codes to compute the dust opacity and for very useful discussions on dust properties. Nuria Calvet and Lee Hartmann for interesting comments and discussions. This work was partly supported by ASI grant ARS 1/R/27/00 and ARS-1/R/073/01 to the Osservatorio di Arcetri. 


\section{References}

Beckwith, S. V. W., Henning, T., \& Nakagawa, Y. 2000, in Protostars and Planets IV, ed. V. Mannings, A. P. Boss, \& S. S. Russell (Tucson: University of Arizona Press), 559

Beckwith, S. V. W., \& Sargent, A. I. 1991, ApJ, 382, L31

Bouwman, J., de Koter, A., van den Ancker, M. E., \& Waters, L. B. F. M. 2000, A\&A, 360, 213

Calvet, N., D’Alessio, P., Hartmann, L., et al. 2002, ApJ, 568, 1008

Chiang, E. I., \& Goldreich, P. 1997, ApJ, 490, 368

Chiang, E. I., Joung, M. K., Creech-Eakman, M. J., et al. 2001, ApJ, 547,1077

Dullemond, C. P., Dominik, C., \& Natta, A. 2001, ApJ, 560, 957

Dullemond, C. P., \& Natta, A. 2003, A\&A, 405, 597

Dunkin, S. K., \& Crawford, I. A. 1998, MNRAS, 288, 275

Dutrey, A., Guilloteau, S., Duvert, G., et al. 1996, A\&A, 309, 493

Felli, M., \& Panagia, N. 1981, A\&A, 102, 424

Kitamura, Y., Momose, M., Yokogawa, S., et al. 2002, ApJ, 581, 357

Krügel, E., \& Siebenmorgen, R. 1994, A\&A, 288, 929

Mannings, V. 1994, MNRAS, 271, 587

Mannings, V., \& Sargent, A. I. 1997, ApJ, 490, 792

Mannings, V., \& Sargent, A. I. 2000, ApJ, 529, 391

Miyake, K., \& Nakagawa, Y. 1993, Icarus, 106, 20
Natta, A., Grinin, V. P., \& Mannings, V. 2000, in Protostars and Planets IV, ed. V. Mannings, A. P. Boss, \& S. S. Russell (Tucson: University of Arizona Press), 559

Palla, F., \& Stahler, S. W. 1993, ApJ, 418, 414

Piétu, V., Dutrey, A., \& Kahane, C. 2003, A\&A, 398, 565

Pollack, J. B., Hollenbach, D., Beckwith, S., et al. 1994, ApJ, 421, 615

Semenov, D., Henning, Th., Helling, Ch., Ilgner, M., \& Sedlmayr, E. 2003, A\&A, 410, 611

Sylvester, R. J., Skinner, C. J., Barlow, M. J., \& Mannings, V. 1996, MNRAS, 279, 915

Testi, L., Natta, A., Shepherd, D. S., \& Wilner, D. J. 2001, ApJ, 554, 1087

Testi, L., Natta, A., Shepherd, D. S., \& Wilner, D. J. 2003, A\&A, 403, 323

Testi, L., Natta, A., Neri, R., Shepherd, D. S., \& Wilner, D. J. 2004, in preparation

Weidenschilling, S. J. 1997, Icarus, 127, 290

Weidenschilling, S. J. 2000, SSRv, 92, 295

Weintraub, D. A., Sandell, G., \& Duncan, W. 1989, ApJ, 340, L69

Wilner, D. J., Bourke, T. L., Wright, C. M., et al. 2003, ApJ, in press

Wilner, D. J., Ho, P. T. P., Kastner, J. H., \& Rodríguez, L. F. 2000, ApJ, 534, L101 